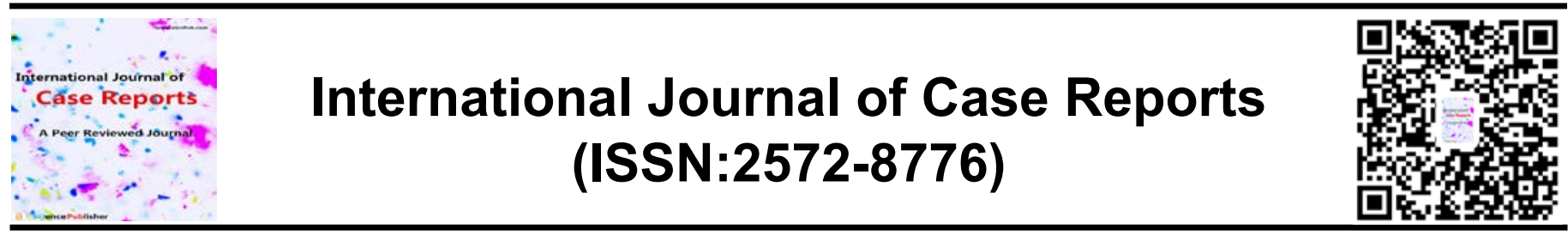

\title{
Natural orifice endoscopic drainage of a perforated duodenal ulcer
}

\author{
Mark Scaife MD, Thomas J Melvin MD, Harry W Sell MD, Kurt R Stahlfeld MD \\ UPMC Mercy Department of Surgery 1400 Locust St Pittsburgh, PA 15219
}

\begin{abstract}
Posterior perforation of gastric and duodenal ulcers is uncomexam findings. The need for surgical treatment has recently UPMC Mercy been questioned in patients with contained perforations due to the success of nonoperative management and the morbidity and mortality associated with surgical intervention.

We present a clinically stable 65-year-old morbidly obese fe-

male transferred to our institution with radiographic evidence of posterior perforation of a duodenal ulcer with a large associated abscess cavity. A fenestrated 9.5 French nasal feeding tube was directed over a guide-wire into the abscess cavity under endoscopic/fluoroscopic guidance and used for decompression. The patient was managed conservatively and repeat computed tomography (CT) demonstrated resolution of the cavity. She was discharged to home after an uncomplicated 10-day hospital course.

Contained posterior peptic/duodenal ulcer perforations associated with an abscess cavity can be successfully managed conservatively in patients that are hemodynamically stable and without overt signs of peritonitis. Natural orifice drainage of the cavity, gastric decompression, antibiotics, PPI administration, and nutritional support can avoid the morbidity and mortality associated with traditional surgical management.
\end{abstract}

*Correspondence to Author:

mon and frequently misdiagnosed due to nonspecific clinical Kurt R Stahlfeld, MD

Department of Surgery

1400 Locust St

Pittsburgh, PA 15219 Phone: 412-232-8097

Email: stahlfeldk @ upmc.edu

How to cite this article:

Mark Scaife, Thomas J Melvin, Harry W Sell, Kurt R Stahlfeld. Natural orifice endoscopic drainage of a perforated duodenal ulcer. International Journal of Case Reports, $20183: 31$

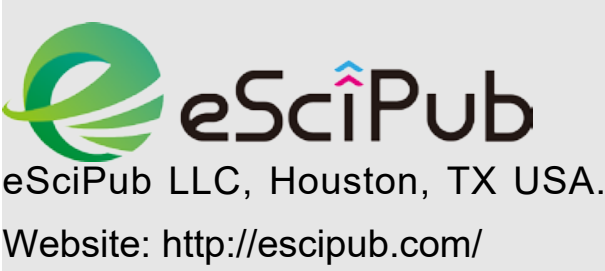

Key Words: Natural orifice, Endoscopy, Peptic ulcer, Duodenal ulcer, Perforation, Posterior Perforation 


\section{Introduction:}

Posterior perforation of a gastric or duodenal ulcer is an uncommon clinical entity complicated by a delay in diagnosis secondary to nonspecific symptomatology [1]. Operative repair in these patients is associated with a high morbidity and mortality [1]. Although uncommon in the United States, selective conservative management of contained anterior duodenal perforations with intravenous fluids, nasogastric decompression, and antibiotics has a success rate of $50-70 \%[2,3]$. In patients who are candidates for non-surgical treatment, spontaneous healing and a well-defined abscess cavity are associated with a favorable outcome [1].

In lieu of percutaneous drainage, natural orifice and naso-biliary drainage has been used to successfully manage abscesses associated with foregut perforations $[1,4,5]$. Choice of these techniques is influenced by body habitus along with the size and chronicity of the abscess cavity. We present a case of a large abscess cavity due to a posterior duodenal ulcer perforation that was managed with a naso-duodenal drainage catheter placed under endoscopic and fluoroscopic guidance.

\section{Case Report:}

A 65 year old morbidly obese female with a history of COPD, chronic NSAID use and prior cholecystectomy presented to a community hospital with three days of nausea, vomiting, and abdominal pain. A CT scan followed by an esophagogastroduodenoscopy

(EGD)

suggested a posterior perforation of the first portion of the duodenum with an associated abscess cavity, prompting transfer to our institution.

Upon arrival, the patient's vital signs were unremarkable aside from mild tachycardia, her abdominal exam was benign, and her leukocytosis had resolved. A repeat CT scan with PO contrast was obtained, which demonstrated extraluminal extravasation into a $10 \mathrm{~cm}$ debris filled abscess cavity in the omental bursa (Figure 1). A subsequent EGD confirmed a large posterior perforation in the first portion of the duodenum (Figure 2). The endoscope was then introduced into the abscess cavity, necrotic tissue was aspirated, and insufflation confirmed no evidence of free perforation. At the time, the therapeutic options being considered were open surgery, percutaneous drainage, and natural orifice drainage. Given the chronic, well-encapsulated appearance of the abscess cavity and the patient's fairly benign clinical status, the decision was made to treat this patient conservatively. Under direct endoscopic visualization a guide-wire was directed into the abscess cavity, after which a 9.5 French fenestrated feeding tube was advanced over the wire under fluoroscopic guidance (Figure 3). The patient was returned to the general medical-surgical floor and managed conservatively with antibiotics, PPI, nasoabscess drainage with low continuous suction, and parenteral nutrition. A follow-up CT scan demonstrated resolution of the abscess cavity and the patient was discharged on hospital day 10 , tolerating a regular diet.

\section{Discussion:}

Posterior perforation of peptic/duodenal ulcers continues to present a diagnostic and therapeutic challenge. Because direct surgical repair is associated with significant morbidity and mortality, alternative non-operative management strategies are being attempted. Conservative management in combination with gastric decompression in the treatment of peptic ulcer perforation was initially proposed by Wangensteen in 1935 [6]. Subsequent retrospective and prospective studies have documented successful non-operative management rates of $50-82 \%$ in select patients $[2,7,8]$. Predictors of failure of conservative management include larger amounts of pneumoperitoneum, tachycardia, pain on digital rectal exam, and increasing age [9].

The initial conservative management strategies focused on gastric decompression in addition to antibiotics. When the perforation is associated 


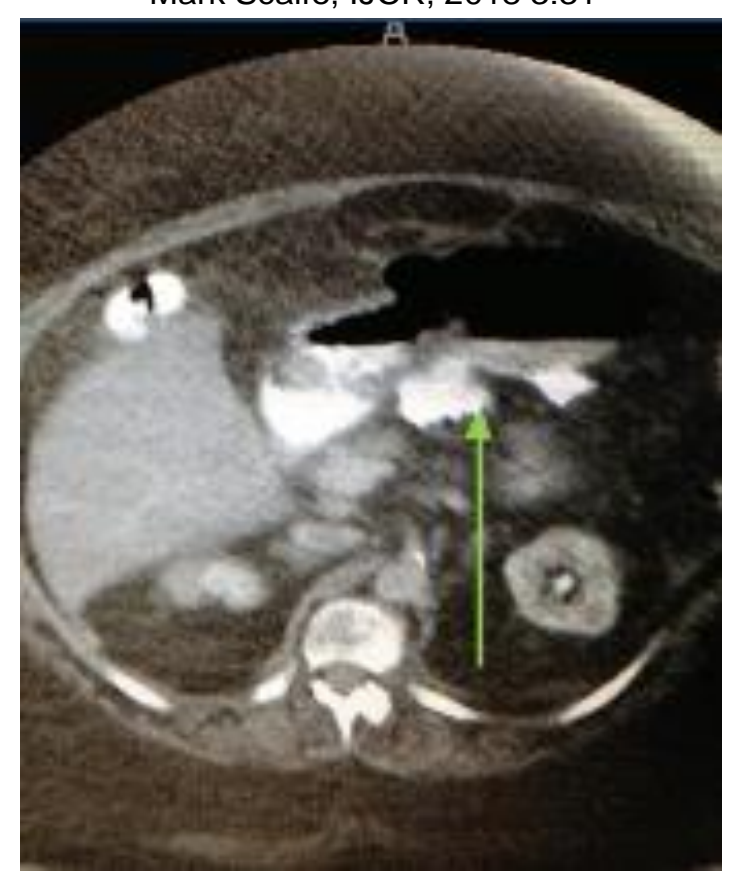

Figure 1: CT showing extraluminal contrast into omental bursa

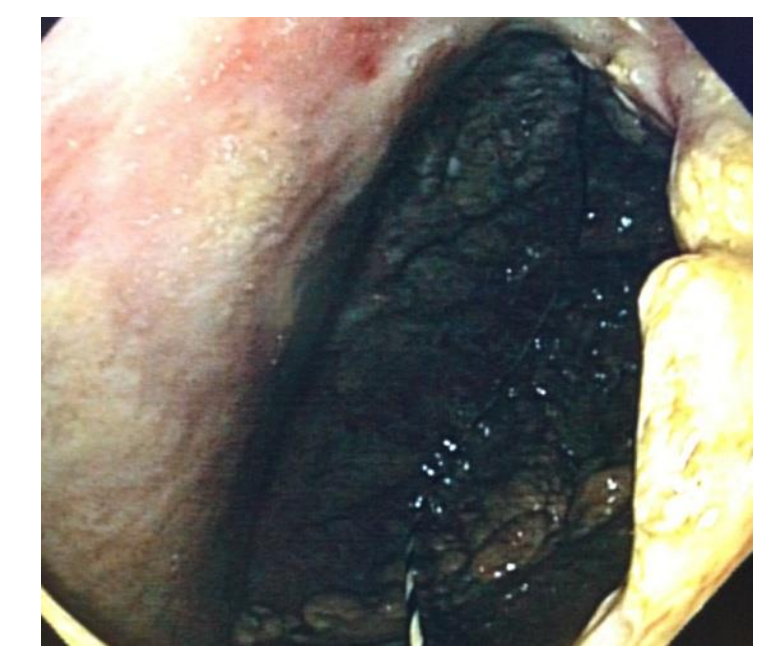

Figure 2: Endoscopic view of large perforated duodenal ulcer

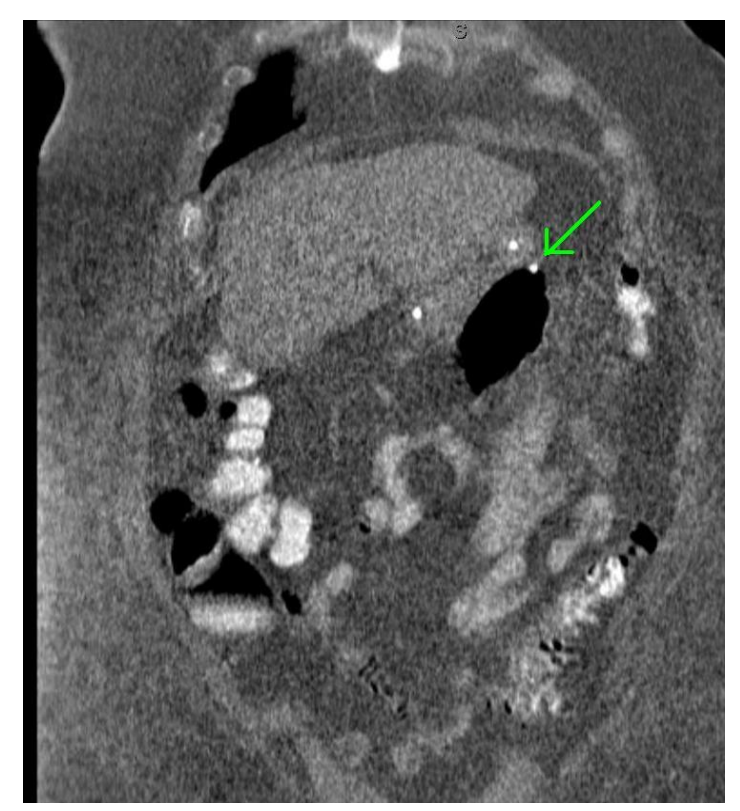

Figure 3: CT showing trans-nasal feeding tube in abscess cavity 
with an abscess cavity, an increasing focus is placed on draining the cavity in addition to decompressing the stomach. Frequently, this is accomplished percutaneously, which places the patient at risk for developing a fistula as well as subjecting them to the discomfort of an external catheter. A novel therapy using an endoluminal vacuum system has been proposed to drain the cavity, but this method needs to be validated. However, using a natural orifice to introduce a catheter that both drains the cavity and decompresses the stomach appears to be a feasible option.

\section{Conclusion:}

In hemodynamically stable patients with no evidence of peritonitis, natural orifice drainage of an abscess cavity associated with peptic ulcer perforation is an acceptable alternative to percutaneous drainage or open surgery. Multidisciplinary teams with access to advanced endoscopic techniques and imaging may use a conservative approach in managing patients with contained foregut perforations.

\section{References:}

1. Wong $\mathrm{CH}$, Chow PK, Ong HS, Chan WH, Khin LW, Soo KC. Posterior perforation of peptic ulcers: presentation and outcome of an uncommon surgical emergency. Surgery 2004;1 35(3):321-5
2. Songne B, Jean F, Foulatier O, Khalil H, Scotté $M$. Non operative treatment for perforated peptic ulcer: results of a prospective study. Ann Chir 2004; 129(10):578-82.

3. Crofts TJ, Park KG, Steele RJ, Chung SS, Li AK. A randomized trial of nonoperative treatment for perforated peptic ulcer. N Engl J Med 1989; 320(15):970-3

4. Xu QY, Yin GW, Chen SX, Jiang F, Bai XJ, Wu JD. Fluoroscopically guided nose tube drainage of mediastinal abscesses in post-operative gastro-eosophageal anastomotic leakage. $\mathrm{Br} \mathrm{J}$ Radiol 2012;85(1019):1477-81

5. Sharma BC, Kumar R, Agarwal N, Sarin SK. Endoscopic biliary drainage by nasobiliary drain or by stent placement in patients with acute cholangitis. Endoscopy 2005;37(5):439-43

6. Wangensteen $O$. Non-operative treatment of localized perforations of the duodenum. Minn Med 1935;18:477

7. Taylor H. Perforated peptic ulcer; treated without operation. Lancet 1946 ;2(6422):441-4

8. Crofts TJ, Park KG, Steele RJ, Chung SS, Li AK. A randomized trial of nonoperative treatment for perforated peptic ulcer. N Engl J Med 1989 ;320(15)970-3

9. Saber A, Gad MA, Ellabban GM. Perforated duodenal ulcer in high risk patients: is percutaneous drainage justified? N Am J Med Sci. $2012 ; 4(1): 35-9$

10. Smallwood NR et al. The use of endoluminal vacuum $(\mathrm{E}-\mathrm{Vac})$ therapy in the management of upper gastrointestinal leaks and perforations. Surg Endo 2016; 30(6): 2473-80

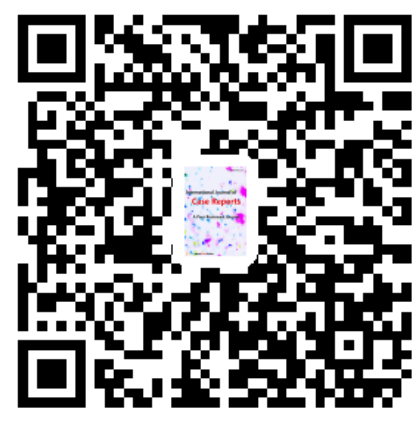

